\title{
Fracture parameter analysis of flat shells under out-of-plane loading using ordinary state-based peridynamics
}

\author{
Ming-Jyun Dai ${ }^{\mathrm{a}}$, Satoyuki Tanaka ${ }^{\mathrm{a}, *}$, Tinh Quoc Bui ${ }^{\mathrm{b}}$, \\ Selda Oterkus ${ }^{\mathrm{c}}$, Erkan Oterkus $^{\mathrm{c}}$ \\ ${ }^{a}$ Graduate School of Advanced Science and Engineering, \\ Hiroshima University, Japan \\ ${ }^{b}$ Department of Civil and Environmental Engineering, \\ Tokyo Institute of Technology, Japan \\ ${ }^{c}$ Department of Naval Architecture, Ocean and Marine Engineering, \\ University of Strathclyde, United Kingdom
}

\begin{abstract}
The present paper is devoted to numerical investigation on fracture parameters of cracked shells subjected to out-of-plane loading using ordinary statebased peridynamics (PD). The nonlocal deformation gradient and equivalent domain integral are introduced to evaluate fracture parameters. To reduce the surface effect and obtain more accurate results, the energy method and volume correction algorithm are considered. Meanwhile, the adaptive dynamic relaxation technique is employed to obtain steady-state solutions. From comparisons between PD results and reference solutions, the proposed PD shell model successfully evaluates fracture parameters in both single- and mixed-mode loading conditions.
\end{abstract}

Keywords: Peridynamics; Nonlocal deformation gradient; Equivalent domain integral; Fracture parameters; Mindlin plate

${ }^{*}$ Corresponding author. E-mail addresses: mingjyundai@gmail.com (M.J. Dai), satoyuki@hiroshima-u.ac.jp (S. Tanaka), bui.t.aa@m.titech.ac.jp (T.Q. Bui), selda.oterkus@strath.ac.uk (S. Oterkus), erkan.oterkus@strath.ac.uk (E. Oterkus) 


\section{Introduction}

A novel numerical method, peridynamics (PD), was proposed by Silling $[1,2]$ to deal with complicated fracture phenomena. The PD theory based on integro-differential equations can inherently define discontinuities in the analysis domain without special numerical techniques. It has been demonstrated that the PD theory successfully investigates different kinds of fracture mechanics problems in several references, such as crack nucleation [3], crack propagation [4], crack branching [5], and crack arrest [6].

Thin-walled structures, e.g., plates and shells, are widely used in engineering applications owing to their high strength-to-weight ratio. Up to now, two-dimensional (2D) plane and three-dimensional (3D) solid models draw the main attention in most PD studies. 3D solid models typically demand a relatively fine discretization in the through-thickness direction to capture an accurate representation of the resistance to bending in thin-walled structures. When simulating thin-walled structures, the computational requirement would significantly increase by 3D solid models. Thus, structural idealization is an effective approach to reduce computational time. Both Kirchhoff-Love [7,8] and Mindlin-Reissner [9-11] plate theories are common approaches for structural idealization in the PD literature.

In fracture mechanics analysis, J-integral and stress intensity factors (SIFs) are regarded as primary fracture parameters in most previous experiments and numerical studies. Generally, the shell model consists of plane stress and plate bending approximations. Stress intensity factors and moment/shear intensity factors correspond to fracture parameters for in-plane and out-of-plane loadings, respectively. Even though out-of-plane loading is one of the major loads in plate and shell structures, its studies are relatively less than in-plane ones. Sosa and Eischen [12] derived the contour integral to evaluate mode-I moment intensity factors. Sosa and Herrmann [13] further employed the contour integral to investigate mixed-mode fracture problems under out-of-plane loading. Zehnder and Viz [14] summarized fracture mechanics phenomena of plates and shells between different computational methods. Several numerical methods were adopted to evaluate moment/shear intensity factors in previous references, such as dual boundary element method (DBEM) [15], extended finite element method (XFEM) $[16,17]$, phantom-node method [18], extended isogeometric analysis (XIGA) $[19,20]$, and meshfree method $[21,22]$.

So far, a few fracture parameter studies have been carried out in the 
PD literature. In the beginning, Silling and Lehoucq [23] developed the state-based PD J-integral. Hu et al. [24] derived the PD J-integral in bondbased peridynamics (BBPD) and compared J-integral results between PD and FEM. Le and Bobaru [25] investigated the effectiveness of surface effect corrections in the PD J-integral evaluation. Stenström and Eriksson [26] presented the alternative J-integral approach via displacements obtained from BBPD. These studies mentioned above were investigated under pure mode-I fracture conditions. Subsequently, the PD studies on mixed-mode fracture conditions were published for dynamic SIFs evaluation [27] and fatigue crack growth analysis [28]. To the authors' knowledge, fracture parameter assessment, e.g., SIFs and J-integral, under out-of-plane loading is still not discussed in the PD framework.

The fracture parameter assessment of flat shell structures is implemented by employing the ordinary state-based peridynamics (OSPD) shell model [10]. The energy method [29] and adaptive dynamic relaxation (ADR) technique [30] are applied to deal with surface effect corrections and quasi-static problems, respectively. The domain form of the J-integral, also known as the equivalent domain integral (EDI), [16] is proposed to evaluate fracture parameters. In addition, the derivative of variables in the J-integral evaluation is obtained by introducing the nonlocal deformation gradient [31]. The presented PD shell model had successfully evaluated fracture parameters under in-plane loading [32]. It aims to assess fracture parameters of cracked shells subjected to out-of-plane loading in the present paper.

The remainder of this paper is organized as follows. The PD shell model and some numerical techniques are briefly introduced in Section 2. The nonlocal deformation gradient and EDI are presented to evaluate fracture parameters in Section 3. Several numerical problems are demonstrated in Section 4. The conclusions are given in Section 5.

\section{Ordinary state-based peridynamic formulation}

A novel non-local theory, Peridynamics, has been rapidly developed in the field of computational mechanics. In the non-local theory, any material point $\boldsymbol{x}$ interacts with the other material points $\boldsymbol{x}^{\prime}$ within a distance $\delta$, as illustrated in Fig. 1. Those material points $\boldsymbol{x}^{\prime}$ are called the family of $\boldsymbol{x}, H_{x}$. In contrast to classical continuum mechanics, the PD equation of motion is expressed in terms of integro-differential equations $[2,30]$ as follows: 


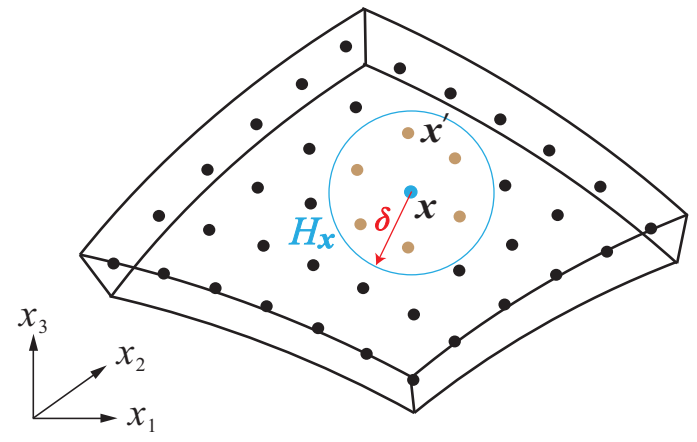

\section{Peridynamics}

Figure 1: The interactional relationship of material points in PD.

$$
\begin{aligned}
\rho(\boldsymbol{x}) \ddot{\boldsymbol{u}}(\boldsymbol{x}, t)=\int_{H_{x}}\left[\boldsymbol{t}\left(\boldsymbol{u}^{\prime}-\boldsymbol{u}, \boldsymbol{x}^{\prime}-\boldsymbol{x}, t\right)\right. \\
\left.\quad-\boldsymbol{t}^{\prime}\left(\boldsymbol{u}-\boldsymbol{u}^{\prime}, \boldsymbol{x}-\boldsymbol{x}^{\prime}, t\right)\right] d V^{\prime}+\boldsymbol{b}(\boldsymbol{x}, t),
\end{aligned}
$$

where $\boldsymbol{x}, \boldsymbol{u}$ and $t$ denote the reference position vector, displacement vector and time, respectively. $\rho$ is the mass density, and $V$ is the volume of a material point. $\ddot{\boldsymbol{u}}, \boldsymbol{t}$, and $\boldsymbol{b}$ represent the acceleration vector, force density vector, and body force density vector, respectively.

Although the PD theory can well solve complicated fracture problems, its low computational efficiency is an inherent drawback. Due to the nonlocal characteristic, the interactions of each material point within $H_{x}$ need to be concerned in every time step. On the other hand, the PD equations of motion are in dynamic form. The iteration procedure is required to obtain steady-state solutions for static problems. Those features lead to expensive computational time in PD. To reduce computational time, the PD shell model proposed in Ref. [10] is adopted to deal with fracture parameter evaluation for thin-walled structures instead of the 3D solid model.

\subsection{Peridynamic model for shell structures}

The OSPD model for shell structures [10] is employed in the present paper. The schematic illustration of the PD shell model is presented in 
Fig. 2. $\boldsymbol{x}_{(k)}$ and $\boldsymbol{y}_{(k)}$ denote the initial and deformed positions of point $k$, respectively. $\varphi$ represents the angle of interaction between points $k$ and $j$ with respect to the $x_{1}$-axis. $\boldsymbol{u}_{(k)}=\left[\begin{array}{lllll}u & v & w & \theta_{x} & \theta_{y}\end{array}\right]^{T}$ and $\boldsymbol{t}_{(k)(j)}$ denote the displacement and force density vectors of point $k$, respectively. Note that the small deformation assumption is considered in the proposed PD model.

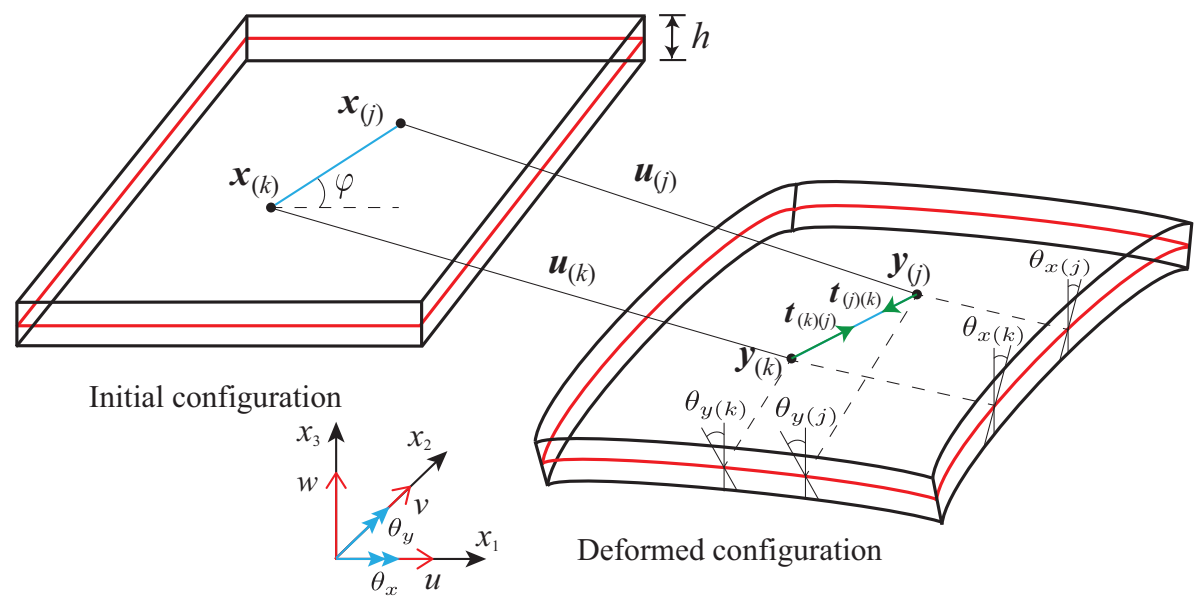

Figure 2: Initial and deformed states of a flat shell in PD [32].

In order to derive the equations of motion for the PD shell model, the strain energy densities in PD for in-plane, bending, and shear deformation components need to be established beforehand. Meanwhile, the dilatations and bond stretches are defined in a similar manner to the Mindlin plate theory. By comparing dilatations and strain energy densities between classical continuum mechanics and PD, the PD constants can be obtained.

The strain energy density in PD for in-plane deformation can be defined as:

$$
\bar{W}_{i p(k)}^{P D}=a_{i p} \vartheta_{i p(k)}^{2}+b_{i p} \sum_{j=1}^{N} s_{i p(k)(j)}^{2} \xi V_{(j)},
$$

where $\xi$ is the distance between $\boldsymbol{x}_{(k)}$ and $\boldsymbol{x}_{(j)} . s_{i p(k)(j)}$ and $\vartheta_{i p(k)}$ represent the relative bond stretch and dilatation for in-plane deformation, respectively. $s_{i p(k)(j)}$ and $\vartheta_{i p(k)}$ are expressed as below: 


$$
\begin{gathered}
s_{i p(k)(j)}=\frac{\left(u_{(j)}-u_{(k)}\right) \cos \varphi+\left(v_{(j)}-v_{(k)}\right) \sin \varphi}{\xi}, \\
\vartheta_{i p(k)}=d_{i p} \sum_{j=1}^{N} s_{i p(k)(j)} V_{(j)} .
\end{gathered}
$$

The PD constants for in-plane deformation are presented as:

$$
a_{i p}=\frac{E h(3 \nu-1)}{4\left(1-\nu^{2}\right)}, \quad b_{i p}=\frac{2}{\pi h \delta^{2}}, \quad d_{i p}=\frac{3 E}{\pi \delta^{3}(1+\nu)},
$$

where $E$ and $\nu$ denote Young's modulus and Poisson's ratio, respectively. $\delta$ is the horizon size, and $h$ is the shell thickness.

The strain energy density in PD for bending deformation can be defined as:

$$
\bar{W}_{b(k)}^{P D}=a_{b} \vartheta_{b(k)}^{2}+b_{b} \sum_{j=1}^{N} s_{b(k)(j)}^{2} \xi V_{(j)},
$$

where $s_{b(k)(j)}$ and $\vartheta_{b(k)}$ represent the relative bond stretch and dilatation for bending deformation, respectively. $s_{b(k)(j)}$ and $\vartheta_{b(k)}$ are expressed as below:

$$
\begin{gathered}
s_{b(k)(j)}=\frac{-\left(\theta_{y(j)}-\theta_{y(k)}\right) \cos \varphi+\left(\theta_{x(j)}-\theta_{x(k)}\right) \sin \varphi}{\xi}, \\
\vartheta_{b(k)}=d_{b} \sum_{j=1}^{N} s_{b(k)(j)} V_{(j)} .
\end{gathered}
$$

The PD constants for bending deformation are presented as:

$$
a_{b}=\frac{E h^{3}(3 \nu-1)}{48\left(1-\nu^{2}\right)}, \quad b_{b}=\frac{2}{\pi h \delta^{2}}, \quad d_{b}=\frac{E h^{2}}{4 \pi \delta^{3}(1+\nu)} .
$$


The strain energy density in PD for shear deformation can be defined as:

$$
\bar{W}_{s(k)}^{P D}=\frac{1}{4} C_{s} \sum_{j=1}^{N}\left(\frac{w_{(j)}-w_{(k)}}{\xi}-\frac{\bar{\theta}_{(j)}-\bar{\theta}_{(k)}}{2}\right)^{2} \xi V_{(j)},
$$

where $\bar{\theta}_{(k)}$ and $\bar{\theta}_{(j)}$ represent the rotations with respect to the interaction bond between points $k$ and $j . \bar{\theta}_{(k)}$ and $\bar{\theta}_{(j)}$ are expressed as below:

$$
\begin{gathered}
\bar{\theta}_{(k)}=-\theta_{y(k)} \cos \varphi+\theta_{x(k)} \sin \varphi, \\
\bar{\theta}_{(j)}=-\theta_{y(j)} \cos \varphi+\theta_{x(j)} \sin \varphi .
\end{gathered}
$$

The PD constants for shear deformation are presented as:

$$
C_{s}=\frac{3 k_{s} E}{\pi \delta^{3}(1+\nu)},
$$

where $k_{s}=5 / 6$ denotes the shear correction factor.

The equations of motion are derived based on the Euler-Lagrange equation.

$$
\frac{d}{d t}\left(\frac{\partial \underline{L}}{\partial \dot{q}_{i}}\right)-\frac{\partial \underline{L}}{\partial q_{i}}=0
$$

where $\underline{L}=T-U$ represents the Lagrangian function. $T$ and $U$ denote the kinetic and total potential energies. $q_{i}$ is the degree of freedom, and $\dot{q}_{i}$ is the time derivative of $q_{i}$.

The equation of motion for the PD shell model can be written as:

$$
\boldsymbol{m}_{(k)} \ddot{\boldsymbol{u}}_{(k)}=\sum_{j=1}^{N}\left(\boldsymbol{t}_{(k)(j)}-\boldsymbol{t}_{(j)(k)}\right) V_{(j)}+\boldsymbol{b}_{(k)}=\sum_{j=1}^{N} \boldsymbol{f}_{(k)(j)} V_{(j)}+\boldsymbol{b}_{(k)},
$$

where $N$ is the total number of material points within $\delta$, and $V_{(j)}$ is the volume of point $j . \boldsymbol{m}_{(k)}, \ddot{\boldsymbol{u}}_{(k)}, \boldsymbol{f}_{(k)(j)}$, and $\boldsymbol{b}_{(k)}$ denote the mass matrix, acceleration 
vector, force density vector, and body force density vector, respectively. The details of the equation of motion are given below:

$$
\begin{gathered}
\boldsymbol{m}_{(k)}=\left[\begin{array}{ccccc}
\rho h & 0 & 0 & 0 & 0 \\
0 & \rho h & 0 & 0 & 0 \\
0 & 0 & \rho h & 0 & 0 \\
0 & 0 & 0 & \frac{\rho h^{3}}{12} & 0 \\
0 & 0 & 0 & 0 & \frac{\rho h^{3}}{12}
\end{array}\right], \\
\ddot{\boldsymbol{u}}_{(k)}=\left[\begin{array}{c}
\ddot{u}_{(k)} \\
\ddot{v}_{(k)} \\
\ddot{w}_{(k)} \\
\ddot{\theta}_{x(k)} \\
\ddot{\theta}_{y(k)}
\end{array}\right], \quad \boldsymbol{f}_{(k)(j)}=\left[\begin{array}{c}
f_{(k)(j)}^{u} \\
f_{(k)(j)}^{v} \\
f_{(k)(j)}^{w} \\
f_{(k)(j)}^{\theta_{x}} \\
f_{(k)(j)}^{\theta_{y}}
\end{array}\right], \quad \boldsymbol{b}_{(k)}=\left[\begin{array}{c}
b_{x(k)} \\
b_{y(k)} \\
b_{z(k)} \\
m_{x(k)} \\
m_{y(k)}
\end{array}\right] .
\end{gathered}
$$

The details of the force density vector $\boldsymbol{f}_{(k)(j)}$ for each component are expressed as below:

$$
\begin{gathered}
f_{(k)(j)}^{u}=\left[\frac{2 a_{i p} d_{i p}}{\xi}\left(\vartheta_{i p(k)}+\vartheta_{i p(j)}\right)+4 b_{i p} s_{i p(k)(j)}\right] \cos \varphi \\
f_{(k)(j)}^{v}=\left[\frac{2 a_{i p} d_{i p}}{\xi}\left(\vartheta_{i p(k)}+\vartheta_{i p(j)}\right)+4 b_{i p} s_{i p(k)(j)}\right] \sin \varphi \\
f_{(k)(j)}^{w}=C_{s}\left\{\frac{w_{(j)}-w_{(k)}}{\xi}-\frac{1}{2}\left[-\left(\theta_{y(k)}+\theta_{y(j)}\right) \cos \varphi\right.\right. \\
\left.\left.\quad+\left(\theta_{x(k)}+\theta_{x(j)}\right) \sin \varphi\right]\right\} \\
f_{(k)(j)}^{\theta_{x}}=\left[\frac{2 a_{b} d_{b}}{\xi}\left(\vartheta_{b(k)}+\vartheta_{b(j)}\right)+4 b_{b} s_{b(k)(j)}\right] \sin \varphi \\
+\frac{1}{2} C_{s}\left\{\left(w_{(j)}-w_{(k)}\right) \sin \varphi\right. \\
\left.-\frac{\xi}{2}\left[-\left(\theta_{y(k)}+\theta_{y(j)}\right) \sin \varphi \cos \varphi+\left(\theta_{x(k)}+\theta_{x(j)}\right) \sin ^{2} \varphi\right]\right\}
\end{gathered}
$$




$$
\begin{aligned}
f_{(k)(j)}^{\theta_{y}}= & -\left[\frac{2 a_{b} d_{b}}{\xi}\left(\vartheta_{b(k)}+\vartheta_{b(j)}\right)+4 b_{b} s_{b(k)(j)}\right] \cos \varphi \\
& -\frac{1}{2} C_{s}\left\{\left(w_{(j)}-w_{(k)}\right) \cos \varphi\right. \\
& \left.-\frac{\xi}{2}\left[-\left(\theta_{y(k)}+\theta_{y(j)}\right) \cos ^{2} \varphi+\left(\theta_{x(k)}+\theta_{x(j)}\right) \sin \varphi \cos \varphi\right]\right\} .
\end{aligned}
$$

\subsection{Numerical implementation}

PD constants are computed based on a complete horizon. If the material point is close to the domain boundary, the PD constants are slightly different from ones computed with the complete horizon [25]. This is called the " surface effect" which strongly affects the accuracy of computational results in the PD theory. The energy method [29] is proposed to reduce the surface effect and obtain more accurate results.

In the energy method, the correction factors are established by comparing dilatations and strain energy densities between classical continuum mechanics and PD. The correction factor for dilatation is expressed as below:

$$
D_{(k)}=\frac{\vartheta^{C M}\left(\boldsymbol{x}_{(k)}\right)}{\vartheta^{P D}\left(\boldsymbol{x}_{(k)}\right)}
$$

With the corrected dilatation, the correction factor for strain energy density is expressed as below:

$$
S_{(k)}=\frac{W^{C M}\left(\boldsymbol{x}_{(k)}\right)-a_{l} \vartheta^{P D}\left(\boldsymbol{x}_{(k)}\right)^{2}}{W^{P D}\left(\boldsymbol{x}_{(k)}\right)-a_{l} \vartheta^{P D}\left(\boldsymbol{x}_{(k)}\right)^{2}},
$$

where $a_{l}$ denotes the PD parameter for dilatation. $l=i p$ and $l=b$ represent the PD parameter for in-plane and bending deformations, respectively.

The mean value of the correction factor is taken for each interaction bond between points $k$ and $j$.

$$
\overline{\mathrm{g}}_{D(k)(j) m}=\frac{D_{(k) m}+D_{(j) m}}{2}, \quad \overline{\mathrm{g}}_{S(k)(j) m}=\frac{S_{(k) m}+S_{(j) m}}{2},
$$

where $m=x_{1}, x_{2}$ represent the $x_{1}$ - and $x_{2}$-axis direction, respectively. 
To approximate the correction factor in the arbitrary direction, the principal values of an ellipse are used, as illustrated in Fig. 3.

$$
G_{\beta(k)(j)}=\left(\left[\frac{n_{x_{1}}}{\overline{\mathrm{g}}_{\beta(k)(j) x_{1}}}\right]^{2}+\left[\frac{n_{x_{2}}}{\overline{\mathrm{g}}_{\beta(k)(j) x_{2}}}\right]^{2}\right)^{-\frac{1}{2}},
$$

where $\beta=D$ represents the dilatation term, and $\beta=S$ represents the strain energy density term. $n_{x_{1}}$ and $n_{x_{2}}$ are the $x_{1}$ and $x_{2}$ components of the normal vector $\boldsymbol{n}$, respectively.

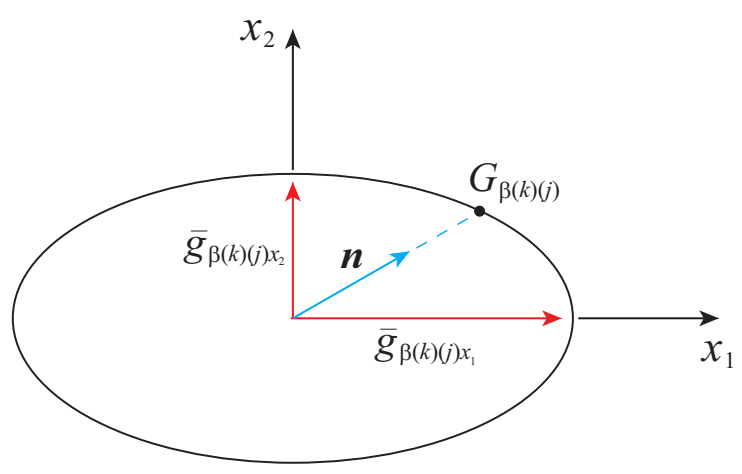

Figure 3: Principal values of an ellipse for the surface corrections.

The material points $\boldsymbol{x}$ in the horizon do not always possess complete volume. Therefore, the volume correction is employed in the PD equation of motion. A complete volume is taken into account when the material point $\boldsymbol{x}$ is located within the range of $\xi<\delta-r$. However, the material point $\boldsymbol{x}$ located within the range of $\delta-r<\xi<\delta$ only possesses a partial volume in the horizon. Thus, a linear volume correction factor is introduced. $r=\Delta x / 2$ is defined. The volume correction factor $v_{(j)}$ is defined as:

$$
v_{(j)}=\left\{\begin{array}{cl}
1, & \xi<\delta-r \\
(\delta+r-\xi) / 2 r, & \delta-r<\xi<\delta \\
0, & \delta<\xi
\end{array}\right.
$$

where $\xi$ is the distance between points $k$ and $j$. 
In order to solve static problems, the ADR technique [30] is adopted in the PD framework. Steady-state solutions can be obtained by introducing stable mass and damping matrices into the $\mathrm{PD}$ equation of motion. The details of the ADR technique for the PD shell model can be found in Ref. [10].

\subsection{Crack modeling}

In the PD theory, the crack segment is modeled by eliminating interaction bonds. When the interaction bond intersects with the crack segment, it is irreversibly broken, as shown in Fig. 4. The dashed line represents the broken bond between points $k$ and $j$. The state of bond interaction is represented by $\mu(\boldsymbol{\xi}, t)$ in Eq. (28) [33].

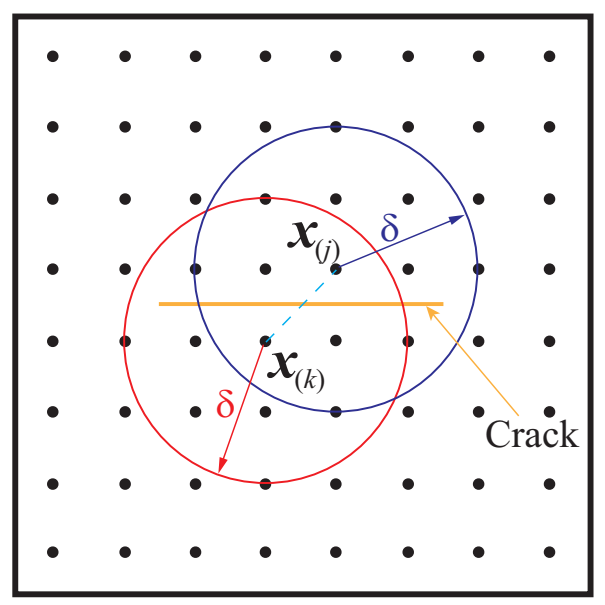

Figure 4: Schematic of crack modeling in PD.

$$
\mu(\boldsymbol{\xi}, t)= \begin{cases}0 & \text { broken bond } \\ 1 & \text { intact bond }\end{cases}
$$

\section{Evaluation of fracture parameters}

Fracture toughness $\left(K_{\mathrm{c}}\right.$ or $\left.J_{\mathrm{c}}\right)$ represents a critical measure of the material resistance to crack propagation. Fracture parameters $(K$ or $J)$ are 
evaluated to determine the status of crack extension. To ensure the safety of structures, fracture parameters should attempt to prevent exceeding fracture toughness. SIFs and J-integral of cracked shells are obtained by employing EDI [16] in the present paper. In the J-integral formulation, the nonlocal deformation gradient [31] is applied to evaluate displacement gradients and stress resultants.

\subsection{Nonlocal deformation gradient}

The nonlocal deformation gradient [2] is commonly used in non-ordinary state-based peridynamics (NOSPD). Thus, NOSPD can easily incorporate with classical constitutive models without reformulating PD force states. However, the nonlocal deformation gradient is only adopted to evaluate displacement gradients and stress resultants in the proposed approach.

First of all, the nonlocal deformation gradient $\boldsymbol{F}$ at $\boldsymbol{x}$ is defined as:

$$
\boldsymbol{F}(\boldsymbol{x})=\left[\int_{H_{x}} \omega(|\boldsymbol{\xi}|)(\underline{\boldsymbol{Y}}(\boldsymbol{\xi}) \otimes \boldsymbol{\xi}) d V^{\prime}\right] \cdot \boldsymbol{K}(\boldsymbol{x}),
$$

where $\boldsymbol{\xi}$ and $\underline{\boldsymbol{Y}}$ denote the reference distance vector and deformation vector state, respectively. $\omega$ is the influence function, and $\boldsymbol{K}$ is the shape tensor. $\boldsymbol{\xi}, \underline{\boldsymbol{Y}}$, and $\boldsymbol{K}$ are given as follows:

$$
\begin{gathered}
\boldsymbol{\xi}=\boldsymbol{x}^{\prime}-\boldsymbol{x}, \\
\underline{\boldsymbol{Y}}(\boldsymbol{\xi})=\left(\boldsymbol{u}^{\prime}+\boldsymbol{x}^{\prime}\right)-(\boldsymbol{u}+\boldsymbol{x}), \\
\boldsymbol{K}(\boldsymbol{x})=\left[\int_{H_{x}} \omega(|\boldsymbol{\xi}|)(\boldsymbol{\xi} \otimes \boldsymbol{\xi}) d V^{\prime}\right]^{-1} .
\end{gathered}
$$

According to the equation $\nabla \boldsymbol{u}=\boldsymbol{F}-\boldsymbol{I}$, the displacement gradient can be obtained. $\boldsymbol{I}$ is the identity matrix. The influence function $\omega=\left(\delta /\left|\boldsymbol{x}_{(j)}-\boldsymbol{x}_{(k)}\right|\right)^{2}$ is chosen [11]. The discrete forms of $\nabla \boldsymbol{u}$ and $\boldsymbol{K}$ are expressed as below:

$$
\nabla \boldsymbol{u}_{(k)}=\left[\sum_{j=1}^{N} \mu_{(k)(j)}\left(\frac{\delta}{\left|\boldsymbol{x}_{(j)}-\boldsymbol{x}_{(k)}\right|}\right)^{2}\left(\boldsymbol{u}_{(j)}-\boldsymbol{u}_{(k)}\right) \otimes\left(\boldsymbol{x}_{(j)}-\boldsymbol{x}_{(k)}\right) V_{j}\right] \boldsymbol{K}_{(k)},
$$




$$
\boldsymbol{K}_{(k)}=\left[\sum_{j=1}^{N} \mu_{(k)(j)}\left(\frac{\delta}{\left|\boldsymbol{x}_{(j)}-\boldsymbol{x}_{(k)}\right|}\right)^{2}\left(\boldsymbol{x}_{(j)}-\boldsymbol{x}_{(k)}\right) \otimes\left(\boldsymbol{x}_{(j)}-\boldsymbol{x}_{(k)}\right) V_{j}\right]^{-1},
$$

where the state of bond interaction $\mu_{(k)(j)}$ is introduced in Eqs. (33) and (34).

After obtaining displacement gradients, strain and stress components can be evaluated by introducing strain-displacement and constitutive relations, respectively. Stress resultants, including moments and transverse shear forces, are yielded by integrating stresses through the shell thickness.

\subsection{Equivalent domain integral}

The contour integral in cracked plates subjected to out-of-plane loading is first proposed by Sosa and Eischen [12]. The contour integral based on the Mindlin plate theory is defined in terms of stress resultants as:

$$
J=\oint_{\Gamma}\left[\bar{W} \delta_{1 \beta}-\left(M_{\alpha \beta} \theta_{\alpha, 1}+Q_{\beta} w_{, 1}\right)\right] n_{\beta} d \Gamma,
$$

where $M_{\alpha \beta}$ and $Q_{\beta}$ represent the moment and shear force components, respectively. $\bar{W}$ denotes the strain energy density expressed as below:

$$
\bar{W}=\frac{1}{2}\left[M_{\alpha \beta} \theta_{\alpha, \beta}+Q_{\beta}\left(\theta_{\beta}+w_{, \beta}\right)\right] .
$$

EDI is more advantageous in PD. It is particularly convenient for computing J-integral with an arbitrary crack under uniform particle discretization. Therefore, the contour integral is recast into the equivalent domain form by applying the divergence theorem. Meanwhile, a smooth weight function $q$ is introduced over the domain of interest. The equivalent domain is illustrated in Fig. 5. EDI can be obtained as:

$$
\begin{aligned}
J= & \int_{A}\left[\left(M_{\alpha \beta} \theta_{\alpha, 1}+Q_{\beta} w_{, 1}\right)-\bar{W} \delta_{1 \beta}\right] q_{, \beta} d A \\
& -\int_{C_{+}+C_{-}}\left[M_{\alpha \beta} \theta_{\alpha, 1}+Q_{\beta} w_{, 1}\right] m_{2} q d C,
\end{aligned}
$$




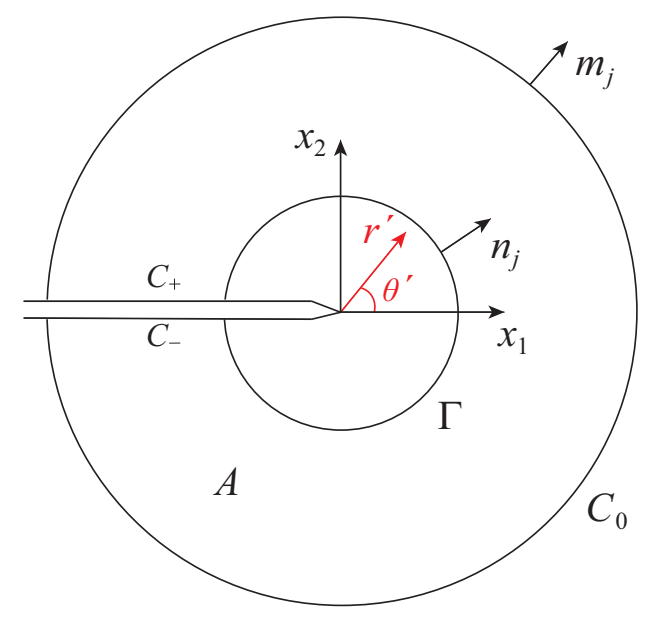

Figure 5: Domain form of the J-integral.

where $C=C_{0}+C_{+}+C_{-}+\Gamma$. The last integral of Eq. (37) omits due to the traction-free crack faces. The traction-free boundary conditions (BCs) are enforced along the upper and lower surfaces of the crack. The details of the traction-free BCs in PD are discussed in Ref. [34]. The discrete form of EDI is expressed as:

$$
J=\sum_{i=1}^{N}\left\{\left[\left(M_{\alpha \beta} \theta_{\alpha, 1}+Q_{\beta} w_{, 1}\right)-\bar{W} \delta_{1 \beta}\right] q_{, \beta}\right\}_{i} A_{i},
$$

where $A_{i}$ is the area of a material point $i$ located within the equivalent domain enclosed by $C$. The weight function $q$ is presented as:

$$
q= \begin{cases}1 & \text { on } \Gamma \\ 0 & \text { on } C_{0} \\ 0<q<1 & C_{0}<r^{\prime}<\Gamma\end{cases}
$$

In linear elastic fracture mechanics, moment intensity factor $K_{1}$ can be directly determined from J-integral under pure mode-I loading. The relationship is expressed as follows:

$$
J=\frac{12 \pi}{E h^{3}} K_{1}^{2}
$$


In mixed-mode loading conditions, the relationship between J-integral and moment/shear intensity factors is given below:

$$
J=\frac{12 \pi}{E h^{3}}\left[K_{1}^{2}+K_{2}^{2}\right]+\frac{\pi}{2 k_{s} G h} K_{3}^{2},
$$

where $G$ is the shear modulus.

The relationship in Eq. (41) is insufficient to separate moment/shear intensity factors. Additional numerical techniques are required to obtain $K_{1}$, $K_{2}$, and $K_{3}$ in mixed-mode loading conditions. Since J-integral can also be regarded as one of the failure criteria, J-integral is chosen to be a fracture parameter for mixed-mode fracture problems in the present paper.

\section{Numerical examples}

The fracture parameter assessment of flat shells is carried out in the PD framework. All the PD results are compared with reference solutions to confirm the effectiveness of the PD shell model. In the numerical examples, uniform particle distribution is utilized and constant horizon size $\delta$ is set to 3.015 $\Delta x . \Delta x$ denotes the particle spacing. Additionally, the normalized

moment intensity factors $F_{1}=K_{1} / M \sqrt{a}$ and $F_{1}=K_{1} / p_{0}\left(\frac{W}{2}\right)^{2} \sqrt{a}$ are defined for bending moment and uniform pressure cases, respectively.

\subsection{Flat square shell with a central crack under uniform pressure}

A flat square shell including a central crack under uniform pressure is analyzed. The length $L$ and width $W$ of the flat shell are $2.0 \mathrm{~mm}$. Three different shell thicknesses are considered, including $W / 2 h=2,6$, and 10. The crack lengths $2 a / W$ are adopted from 0.1 to 0.9 . Young's modulus $E$ and Poisson's ratio $\nu$ are $1,000 \mathrm{GPa}$ and 0.3 , respectively. The flat shell is subjected to uniform pressure $p_{0}=1.0 \mathrm{MPa}$ with simply supported BCs on the top and bottom edges. The numerical model and boundary conditions are shown in Fig. 6. Four different particle spacings are taken, including $\Delta x=0.05,0.025$, 0.0125 , and $0.00625 \mathrm{~mm}$.

At first, the convergence analysis of $K_{1}$ is carried out. From Fig. 7, it can be found that the error of $K_{1}$ gradually decreases with smaller particle spacing. When the particle spacing is less than $0.00625 \mathrm{~mm}$, the error is less than $1 \%$. Thus, $\Delta x=0.00625 \mathrm{~mm}$ is used in the following examination of domain-independence. 

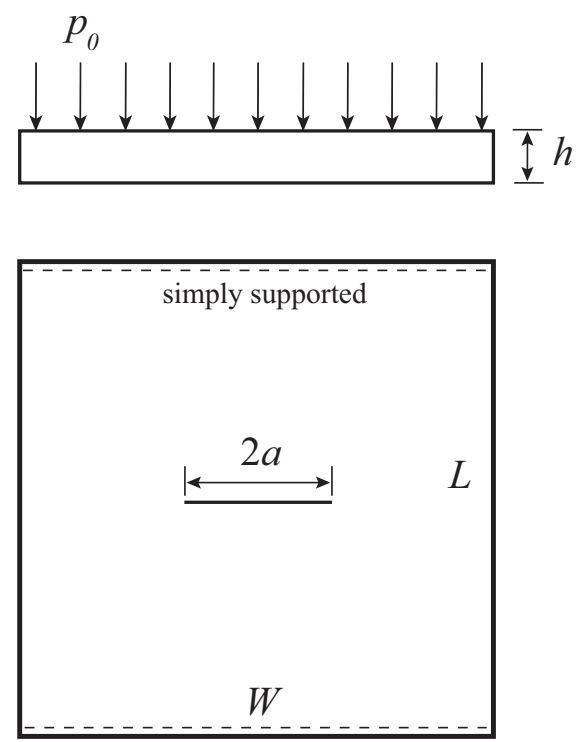

Figure 6: Square plate under uniform pressure with a central crack.

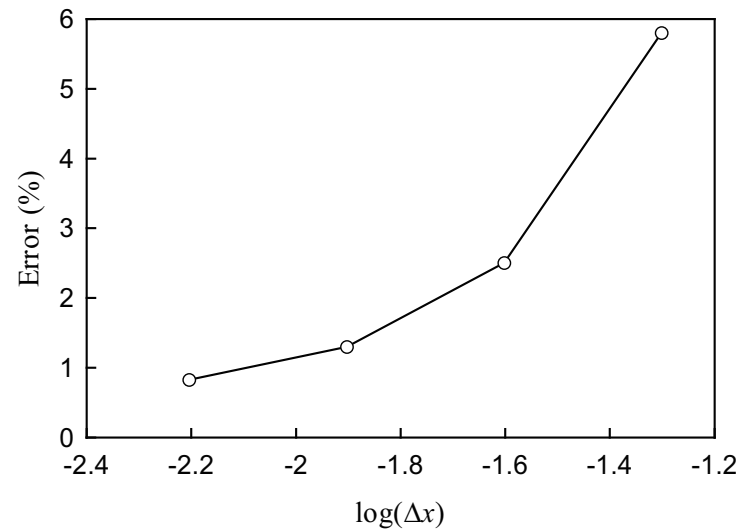

Figure 7: Convergence analysis of $K_{1}$. 
Path-independence and domain-independence of fracture parameters are important features in fracture mechanics for the contour integral and equivalent domain integral, respectively. To examine the domain-independence of $K_{1}$, the normalized $K_{1}$ is assessed for different integral domains with various crack lengths. In the domain-independence assessment, $W / 2 h=2$ is set and five different integral domains are taken. The hollow circle is adopted for integral domains, as shown in Fig. 5. The inner radius of $\Gamma$ is set to $4 \Delta x$. Five outer radii of $C_{0}$ are defined from $6 \Delta x$ to $10 \Delta x$ corresponded to Domain $1-5$, respectively. The normalized $K_{1}$ results are presented in Table 1 . According to the $K_{1}$ results, the domain-independence of $K_{1}$ is confirmed.

Moreover, the normalized $K_{1}$ is also evaluated for three different shell thicknesses with various crack lengths. The PD results are compared with the reference results computed in Ref. [12]. The comparison in Fig. 8 shows that the PD results are in good agreement with the reference results.

Table 1: Normalized moment intensity factor for different crack lengths and integral domains.

\begin{tabular}{ccccccc}
\hline & & \multicolumn{5}{c}{ Domain No. } \\
\cline { 3 - 7 } $2 a / W$ & Ref.[12] & 1 & 2 & 3 & 4 & 5 \\
\hline 0.1 & 0.457 & 0.467 & 0.476 & 0.467 & 0.467 & 0.470 \\
0.2 & 0.428 & 0.430 & 0.439 & 0.444 & 0.438 & 0.437 \\
0.3 & 0.426 & 0.432 & 0.440 & 0.432 & 0.432 & 0.434 \\
0.4 & 0.433 & 0.429 & 0.438 & 0.443 & 0.437 & 0.437 \\
0.5 & 0.452 & 0.460 & 0.469 & 0.460 & 0.460 & 0.463 \\
0.6 & 0.494 & 0.489 & 0.499 & 0.505 & 0.498 & 0.498 \\
0.7 & 0.566 & 0.570 & 0.582 & 0.571 & 0.571 & 0.574 \\
\hline
\end{tabular}

\subsection{Flat rectangular shell with an edge crack under bending load}

A flat rectangular shell including an edge crack under bending load is investigated. The length $L$ and width $W$ of the flat shell are 2.0 and 1.0 $\mathrm{mm}$, respectively. The crack lengths $a / W$ are adopted from 0.1 to 0.9 . Two different shell thicknesses, $W / h=2$ and 10, are considered. $E=210 \mathrm{GPa}$ and $\nu=0.3$ are chosen for the material properties. The flat shell is subjected to bending moments $M=1.0 \mathrm{MPa}-\mathrm{mm}$ with simply supported BCs on the top and bottom edges. The numerical model and boundary conditions are illustrated in Fig. 9. The particle spacing $\Delta x$ is $0.008 \mathrm{~mm}$. 


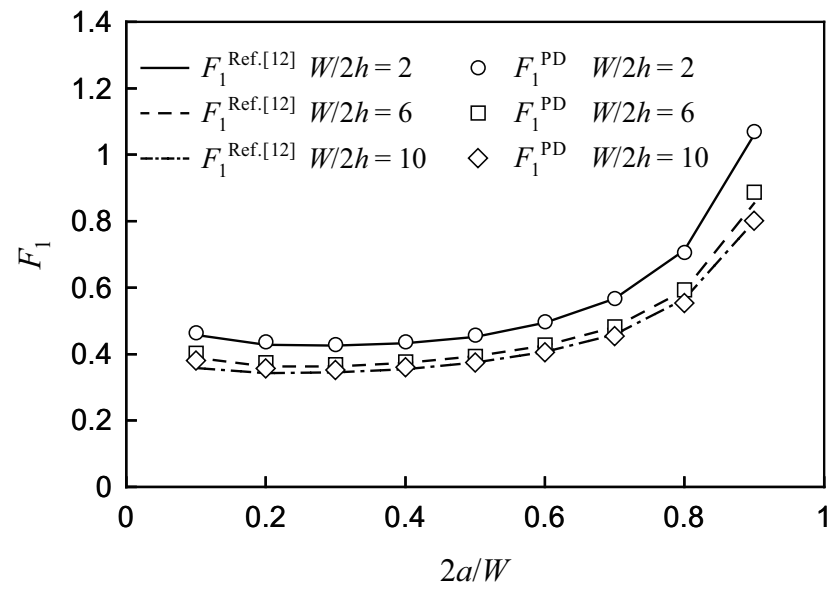

Figure 8: Normalized moment intensity factor for different crack lengths.

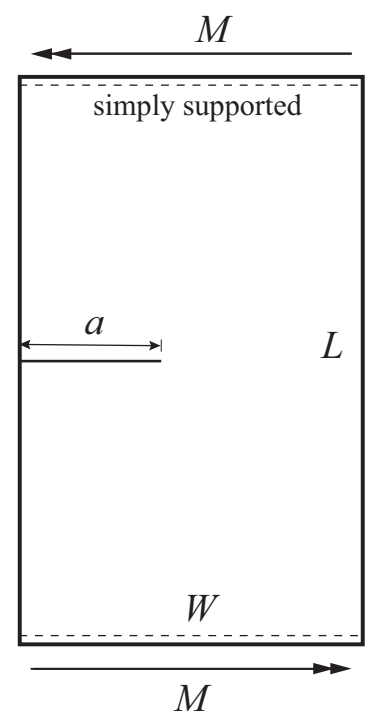

Figure 9: Rectangular plate under bending with an edge crack. 
The normalized $K_{1}$ is investigated for two different shell thicknesses with various crack lengths. In the previous studies, this numerical example had been conducted by using DBEM [15] and RKPM [21]. Both studies are considered as reference solutions to examine the proposed approach. The comparison between three numerical methods is given in Fig. 10. The PD results agree well with the other numerical results. It is worth mentioning that the similar phenomena of $F_{1}$ happen between Fig. 8 and Fig. 10 . $F_{1}$ approximately maintains constant when $a / W$ is less than 0.6 and then significantly increases when $a / W$ is greater than 0.6.

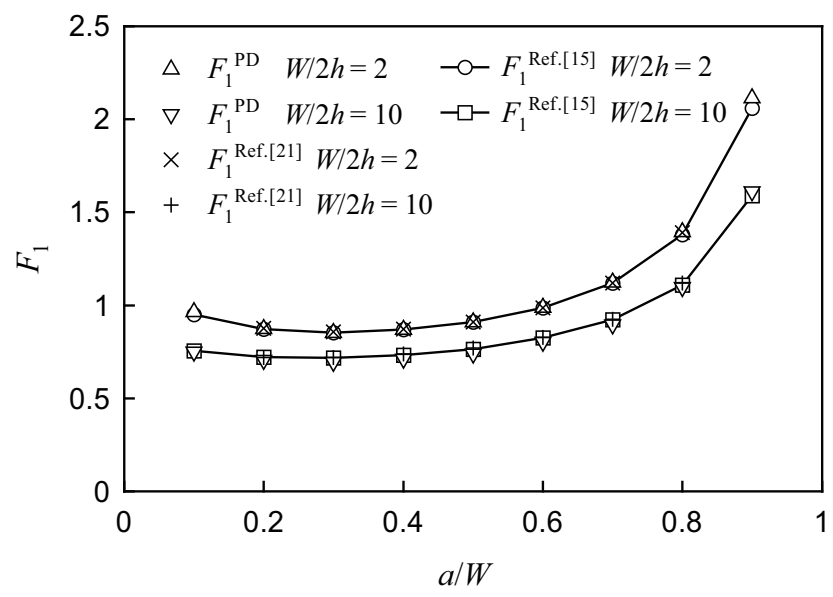

Figure 10: Normalized moment intensity factor for different crack lengths.

\subsection{Flat square shell with symmetric double edge cracks under bending load}

A flat square shell including symmetric double edge cracks under bending load is calculated. The geometry sizes and material properties are the same as the first example, but only two shell thicknesses, $W / 2 h=2$ and 10 , are considered. Two cracks are symmetrically arranged on the left and right edges of the flat shell. The crack lengths $2 c / W$ are adopted from 0.1 to 0.9. The flat shell is subjected to bending moments $M=1.0 \mathrm{MPa}-\mathrm{mm}$ with simply supported BCs on the top and bottom edges. In Fig. 11, the numerical model and boundary conditions are presented. The particle spacing $\Delta x$ is $0.008 \mathrm{~mm}$. 


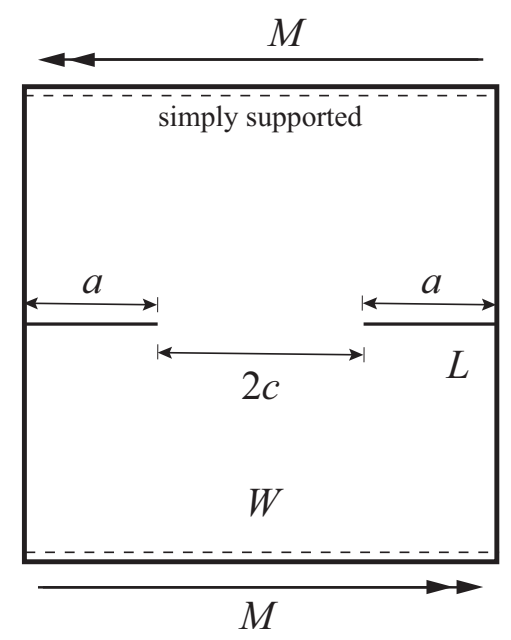

Figure 11: Rectangular plate under bending with symmetric double edge cracks.

The normalized $K_{1}$ is analyzed for two different shell thicknesses with various crack lengths. Sosa and Eischen [12] and Dirgantara and Aliabadi [15] carried out the same numerical example. The $F_{1}$ results of the present method and reference solutions are shown in Fig. 12. The developed PD model offers accurate solutions since its computed values well match the reference ones. From Fig. 12, $F_{1}$ greatly increases with smaller $c$. It means that the fracture parameter of a crack tip is strongly influenced by the other crack tip when the distance between crack tips becomes closer.

\subsection{Flat square shell with an inclined center crack under bending load}

A flat square shell including an inclined center crack under bending load is considered. The length $L$ and width $W$ of the flat shell are $10 \mathrm{~mm}$. The crack length $2 a$ is $1.0 \mathrm{~mm}$, and seven crack angles $\phi$ are adopted from $0^{\circ}$ to $90^{\circ}$. The shell thicknesses $h / a$ are varied from 0.1 to 9.0 at regular intervals of 0.1. $E=200 \mathrm{GPa}$ and $\nu=0.3$ are chosen for the material properties. The boundary conditions presented in Fig. 13 are the same as the third example. The particle spacing $\Delta x$ is $0.04 \mathrm{~mm}$.

The normalized $K_{1}$ is calculated for different shell thicknesses when $\phi=0^{\circ}$. Two reference solutions obtained from Sih [35] and Joseph and Erdogan [36] are available. The PD results are compared with the reference solutions mentioned above. The comparisons of $K_{1}$ for $h / a=0.1-0.9$ and $h / a=1.0-9.0$ 


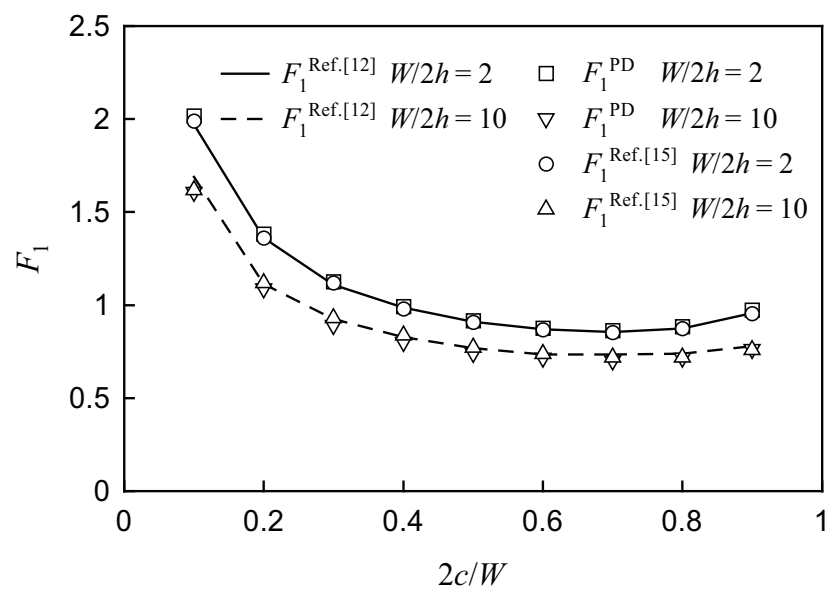

Figure 12: Normalized moment intensity factor for different crack lengths.

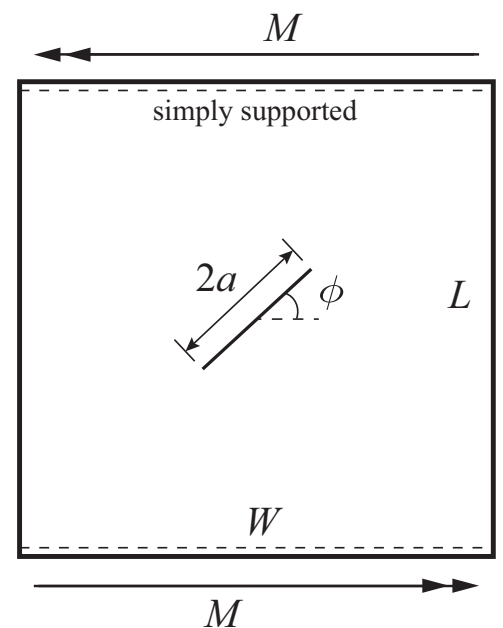

Figure 13: Square plate under bending with an inclined center crack. 
are shown in Fig. 14 (a) and (b), respectively. For thicker shells, the $F_{1}$ results have a good agreement between three different numerical methods. However, the PD results are obviously closer to the results of Ref. [36] for thinner shells. The same conclusion is found in Refs. [17] and [22].

The J-integral is assessed for different crack angles when $h / a=2.0$ in mixed-mode fracture conditions. The reference results of moment/shear intensity factors are taken from Refs. [17] and [35]. By substituting $K_{1}, K_{2}$, and $K_{3}$ into Eq. (41), the reference result of J-integral can be obtained. The comparisons of J-integral for $\nu=0.0$ and 0.3 are expressed in Fig. 15 (a) and (b), respectively. From the J-integral results between PD and reference solutions, it is confirmed that the reasonable $\mathrm{PD}$ results can be obtained in mixed-mode loading conditions by using the proposed approach.

(a)

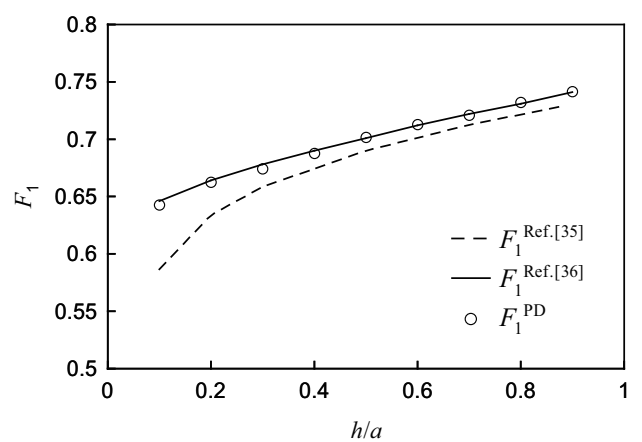

(b)

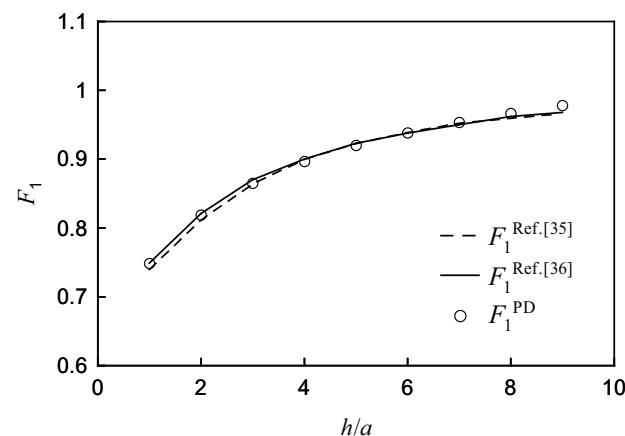

Figure 14: Normalized moment intensity factor for different plate thicknesses at $\phi=0^{\circ}$, (a) $h / a=0.1-0.9$, (b) $h / a=1.0-9.0$.

\section{Conclusion}

In the present paper, the OSPD shell model is proposed to assess fracture parameters under out-of-plane loading. Moment intensity factor and J-integral determine the status of crack extension under pure mode-I loading and mixed-mode loading conditions, respectively. The nonlocal deformation gradient and EDI are applied to evaluate fracture parameters. Some numerical techniques are employed to obtain more accurate solutions in quasi- 
(a)

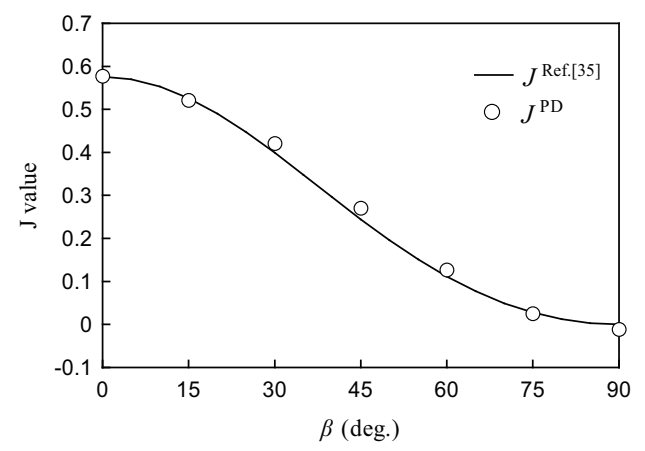

(b)

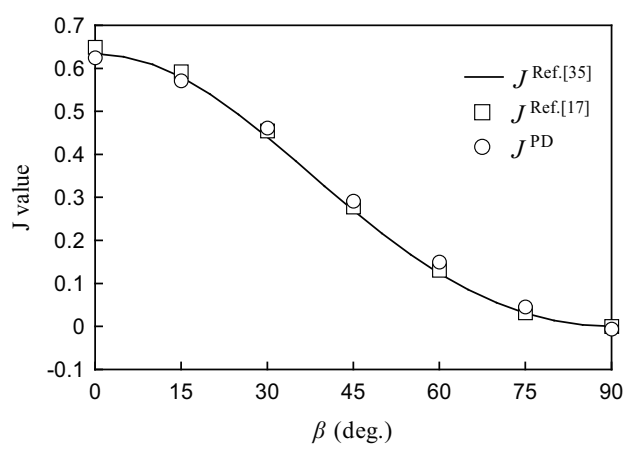

Figure 15: J-integral value for different crack angles, (a) $\nu=0.0$, (b) $\nu=0.3$.

static problems, including the energy method, volume correction, and ADR method.

In order to examine the accuracy and effectiveness of the proposed approach, the PD results are compared with the other reference solutions. In pure mode-I loading conditions, the well accurate PD results of moment intensity factor can be determined. In addition, the reasonable PD solutions of J-integral can also be obtained under mixed-mode loading conditions. It is demonstrated that fracture parameters can be successfully evaluated by the presented PD approach.

\section{Acknowledgements}

The authors gratefully acknowledge financial support from Japan-Taiwan Exchange Association and Sasakawa Scientific Research Grant.

\section{References}

[1] Silling SA. Reformulation of elasticity theory for discontinuities and long-range forces. J Mech Phys Solids 2000;48:175-209.

[2] Silling SA, Epton M, Weckner O, Xu J, Askari E. Peridynamic states and constitutive modeling. J Elast 2007;88:151-184. 
[3] Silling SA, Weckner O, Askari E, Bobaru F. Crack nucleation in a peridynamic solid. Int J Fract 2010;162:219-227.

[4] Agwai A, Guven I, Madenci E. Predicting crack propagation with peridynamics: a comparative study. Int J Fract 2011;171:65-78.

[5] Ha YD, Bobaru F. Studies of dynamic crack propagation and crack branching with peridynamics. Int J Fract 2010;162:229-244.

[6] Imachi M, Tanaka S, Ozdemir M, Bui TQ, Oterkus S, Oterkus E. Dynamic crack arrest analysis by ordinary state-based peridynamics. Int J Fract 2020;221:155-169.

[7] O'Grady J, Foster J. Peridynamic plates and flat shells: A nonordinary, state-based model. Int J Solids Struct 2014;51:4572-4579.

[8] Yang Z, Vazic B, Diyaroglu C, Oterkus E, Oterkus S. A Kirchhoff plate formulation in a state-based peridynamic framework. Math Mech Solids 2020;25:727-738.

[9] Diyaroglu C, Oterkus E, Oterkus S, Madenci E. Peridynamics for bending of beams and plates with transverse shear deformation. Int J Solids Struct 2015;69:152-168.

[10] Nguyen CT, Oterkus S. Peridynamics for the thermomechanical behavior of shell structures. Eng Fract Mech 2019;219:106623.

[11] Nguyen CT, Oterkus S. Investigating the effect of brittle crack propagation on the strength of ship structures by using peridynamics. Ocean Eng 2020;209:107472.

[12] Sosa H, Eischen J. Computation of stress intensity factors for plate bending via a path-independent integral. Eng Fract Mech 1986;25:451462.

[13] Sosa H, Herrmann G. On invariant integrals in the analysis of cracked plates. Int J Fract 1989;40:111-126.

[14] Zhender AT, Viz MJ. Fracture mechanics of thin plates and shells under combined membrane, bending and twisting loads. Appl Mech Rev 2005;58:37-48. 
[15] Dirgantara T, Aliabadi MH. Stress intensity factors for cracks in thin plates. Eng Fract Mech 2002;69:1465-1486.

[16] Dolbow J, Moës N, Belytschko T. Discontinuous enrichment in finite elements with a partition of unity method. Finite Elem Anal Des 2000;35:235-260.

[17] Dolbow J, Moës N, Belytschko T. Modeling fracture in MindlinReissner plates with the extended finite element method. Int J Solids Struct 2000;37:7161-7183.

[18] Chau-Dinh T, Zi G, Lee PS, Rabczuk T, Song JH. Phantom-node method for shell models with arbitrary cracks. Comput Struct 2012;9293:242-256.

[19] Nguyen-Thanh N, Valizadeh N, Nguyen MN, Nguyen-Xuan H, Zhuang X, Areias P, Zi G, Bazilevs Y, De Lorenzis L, Rabczuk T. An extended isogeometric thin shell analysis based on Kirchhoff-Love theory. Comput Methods Appl Mech Engrg 2015;284:265-291.

[20] Yu T, Yuan H, Gu J, Tanaka S, Bui TQ. Error-controlled adaptive LR B-splines XIGA for assessment of fracture parameters in throughcracked Mindlin-Reissner plates. Eng Fract Mech 2020;229:106964.

[21] Tanaka S, Suzuki H, Sadamoto S, Imachi M, Bui TQ. Analysis of cracked shear deformable plates by an effective meshfree plate formulation. Eng Fract Mech 2015;144:142-157.

[22] Tanaka S, Suzuki H, Sadamoto S, Okazawa S, Yu T, Bui TQ. Accurate evaluation of mixed-mode intensity factors of cracked shear-deformable plates by an enriched meshfree Galerkin formulation. Arch Appl Mech 2017;87:279-298.

[23] Silling SA, Lehoucq RB. Peridynamic theory of solid mechanics. Adv Appl Mech 2010;44:73-168.

[24] $\mathrm{Hu}$ W, Ha YD, Bobaru F, Silling SA. The formulation and computation of the nonlocal J-integral in bond-based peridynamics. Int J Fract 2012;176:195-206. 
[25] Le QV, Bobaru F. Surface corrections for peridynamic models in elasticity and fracture. Comput Mech 2018;61:499-518.

[26] Stenström C, Eriksson K. The J-contour integral in peridynamics via displacements. Int J Fract 2019;216:173-183.

[27] Imachi M, Tanaka S, Bui TQ. Mixed-mode dynamic stress intensity factors evaluation using ordinary state-based peridynamics. Theor Appl Fract Mech 2018;93:97-104.

[28] Jung J, Seok J. Mixed-mode fatigue crack growth analysis using peridynamic approach. Int J Fatigue 2017;103:591-603.

[29] Madenci E, Oterkus S. Ordinary state-based peridynamics for plastic deformation according to von Mises yield criteria with isotropic hardening. J Mech Phys Solids 2016;86:192-219.

[30] Madenci E, Oterkus E. Peridynamic theory and its applications. New York: Springer; 2014.

[31] Breitenfeld MS, Geubelle PH, Weckner O, Silling SA. Non-ordinary state-based peridynamic analysis of stationary crack problems. Comput Methods Appl Mech Engrg 2014;272:233-250.

[32] Dai MJ, Tanaka S, Oterkus S, Oterkus E. Mixed-mode stress intensity factors evaluation of flat shells under in-plane loading employing ordinary state-based peridynamics. Theor Appl Fract Mech 2020;accepted.

[33] Silling SA, Askari E. A meshfree method based on the peridynamic model of solid mechanics. Comput Struct 2005;83:1526-1535.

[34] Madenci E, Barut A, Futch M. Peridynamic differential operator and its applications. Comput Methods Appl Mech Engrg 2016;304:408-451.

[35] Sih GC, editor. Mechanics of fracture volume 3: plate and shells with cracks. Leyden: Noordhoff; 1977.

[36] Joseph PF, Erdogan F. Bending of a thin Reissner plate with a through crack. J Appl Mech 1991;58:842-846. 